\title{
Daydreamer and Night Owl: Comparing Positive and Negative Outcome Cases in an Online, Clinician-Guided, Self-Help Intervention for Social Anxiety Disorder
}

\section{AVA SCHULZ, ${ }^{\mathrm{a}, \mathrm{b}}$ ALESSIA VINCENT, ${ }^{\mathrm{a}}$ \& THOMAS BERGER ${ }^{\mathrm{a}}$}

\author{
${ }^{a}$ Department of Clinical Psychology and Psychotherapy, University of Bern, Switzerland \\ b Correspondence regarding this article should be sent to Ava Schulz, Department of Clinical Psychology and \\ Psychotherapy, University of Bern, Fabrikstrasse 8, 3007 Bern, Switzerland \\ Email: ava.schulz@psy.unibe.ch \\ Author Note: This research is supported by a grant from the Swiss National Foundation. \\ Editor Note: The authors were provided the opportunity to respond to the commentaries in this issue about their case \\ studies. However, the authors responded that they did not feel a need to do this since they agreed with almost all the \\ discussion points raised by the commentaries.
}

\begin{abstract}
Internet-Based Cognitive-Behavior Treatment (ICBT) has garnered strong empirical support in the last decade. However, despite the growing body of evidence that web-based treatments work, there are still a considerable number of clients who do not benefit sufficiently from such interventions. Recently, research has started to focus on identifying factors that affect treatment outcome and adherence to Internet interventions. To explore the difference between clients who are successful versus unsuccessful in response to ICBT, this article presents two systematic case studies that describe the course of treatment of a positive-outcome client (named "Daydreamer") and a more negative outcome client (named "Night Owl") in clinician-guided ICBT for Social Anxiety Disorder (SAD). We present focal points of therapist communication, as well as the clients' individual gains and obstacles. Lastly, we explore several factors that seem to be crucial in this specific setting, such as therapist support, motivation, and establishing a working alliance via the Internet.
\end{abstract}

Key words: Social Anxiety Disorder; Internet-Based CBT Treatment (ICBT); cognitive-behavioral treatment; clinician-guided self-help; clinical case studies; case studies

\section{CASE CONTEXT AND METHOD}

\section{Treating Mental Disorders Online}

Since its beginning in the late 1990s, research on Internet-based treatments has skyrocketed. There is a growing body of evidence that interventions delivered via the Internet can effectively treat various mental disorders such as depression, anxiety disorders, and somatic conditions (Andersson, Cuijpers, Carlbring, Riper, \& Hedman, 2014; Andrews, Cuijpers, Craske, P., \& Titov, 2010; Barak, Hen, Boniel-Nissim, \& Shapira, 2008; Klein et al., 2013). Specifically, by now, more than 100 controlled studies evaluating Internet-based treatments in the field of 
anxiety disorders, depression, and other conditions have shown promising results (Schulz et al., 2014). Particularly in the treatment of anxiety disorders, independent replications have shown moderate to large effect sizes in comparison with control groups (Schulz et al., 2014, 2016). Moreover, several studies have found Internet-based treatments to be equally effective compared to traditional psychotherapies.

It is often emphasized that internet-based treatments, such as Internet-Based CognitiveBehavior Treatments (ICBT), are not intended as a replacement for therapy in its traditional setting, but rather as an opportunity to fill the huge supply gap in mental health care (Berger \& Andersson, 2009). As a result of the empirical support, ICBT is already partly included in routine health care in countries such as Australia, Sweden, and the Netherlands (Hedman et al., 2013).

Social Anxiety Disorder (SAD) is the third most common mental disorder after depression and alcohol dependency and is considered the single most prevalent anxiety disorder (Kessler, 2003). Perhaps for this reason, SAD is one of the most researched disorders in the field of ICBT (Boettcher, Carlbring, Renneberg, \& Berger, 2013). People suffering from SAD fear that others might find their behavior or their observable physical reactions (like blushing) embarrassing (Stangier, Heidenreich, \& Peitz, 2003). Only a small percentage of people with SAD receive adequate treatment (Chartier, Hazen, \& Stein, 1998) since the very nature of the disorder can keep people from seeking help in a traditional psychotherapeutic setting. Thus, Internet-delivered interventions with their easy accessibility, anonymity, and flexibility might be especially attractive for people with SAD.

Research consistently suggests that web-based interventions for SAD can significantly improve social anxiety symptoms (Boettcher, Carlbring, et al., 2013). Trials directly comparing ICBT with face-to-face therapy have revealed that both treatment conditions made large and similar improvements (e.g. Andrews, Davies, \& Titov, 2011; Botella et al., 2010; Hedman, Andersson, et al., 2011). Moreover, treatment gains were stable for up to five years after treatment termination (Hedman, Furmark, et al., 2011).

Most of the growing body of evidence comes from studies evaluating Internet-based, clinician-guided, self-help treatments. While patients work their way through a structured selfhelp program comprising several modules or lessons, which are typically based on CBT manuals (i.e., ICBT), therapists or coaches assist and support them via a secured e-mail system. Clinician guidance seems to play an important role in treatment outcome: meta-analyses suggest a superiority of guided versus unguided self-help treatments in terms of adherence to treatment and efficacy (Palmqvist, Carlbring, \& Andersson, 2007; Spek et al., 2007). Although it should be noted that clinician support does not seem necessary in some cases and positive results have also been reported for unguided self-help programs for SAD (Berger et al., 2011; Titov, Andrews, Choi, Schwencke, \& Johnston, 2009). Overall, it can be said that ICBT for SAD produces significant improvements. Nevertheless, far from all participants benefit sufficiently from ICBT. The number of individual participants fulfilling the criterion of "clinically significant change" (Jacobson \& Truax, 1991) - i.e., statistically significant change from a starting point above the clinical cut-off on a SAD measure to an ending point below the clinical cut-off on that 
measure-ranges between 36-56\% across studies on ICBT for SAD (Boettcher, Carlbring, et al., 2013).

\section{The Present Case Studies: A Focus on Differential Outcomes}

The two cases described below—named "Daydreamer" and "Night Owl”-were observed in a randomized controlled trial (RCT), conducted by the authors, of an Internet-Based Cognitive-Behavior Treatment (ICBT) intervention for Social Anxiety Disorder (Schulz, Stolz, \& Berger, 2014; Schulz et al., 2016). In the RCT, 149 individuals with a diagnosis of Social Anxiety Disorder (SAD) were randomly assigned to one of three conditions: ICBT with clinician-guided individual treatment; ICBT with clinician-guided group treatment; and a waiting list control. Using measures of SAD, depression, general psychiatric symptomatology, and interpersonal problems, we found that at post-treatment, both active conditions showed significantly superior outcomes regarding SAD symptoms compared to the waiting list group, and the two active conditions did not differ significantly in symptom reduction. Treatment gains were maintained at follow-up. As specific examples of the positive findings, the effect sizes on the two primary SAD measures for the ICBT with clinician-guided individual treatment condition were 1.50 and 1.08 at post-treatment, and 1.55 and 1.33 at follow-up, respectively.

The Daydreamer and Night Owl cases were both drawn from the clinician-guided individual treatment condition of the RCT. They were selected for qualitative case study because they differed in their outcomes, with Daydreamer having a very positive outcome, and Night Owl having a generally negative outcome. Such consideration of differential outcomes in ICBT for SAD raises the question of why some individuals benefit sufficiently to be considered as cured, whereas others show insufficient or no improvement. In other words, if ICBT works, how exactly does change happen, and for whom?

The knowledge about mechanisms of change in ICBT, as well as predictors or moderators of outcome, is still scarce (Andersson, Carlbring, Berger, Almlöv, \& Cuijpers, 2009). However, some studies have found correlations between certain predictors and outcome indicators. Boettcher, Renneberg, \& Berger, 2013; Tillfors, Furmark, Carlbring, \& Andersson, 2015). A particularly useful example of a recent correlational study is a recent individual patient data meta-analysis by Karyotaki and colleagues (2015). These investigators found that male gender, lower educational level, and comorbid anxiety disorders increase the risk of dropping out in unguided self-help programs for depression. Further research is needed to replicate these findings in diverse patient populations (e.g., for anxiety disorders) or for other forms of ICBT (e.g., clinician-guided self-help). Regarding mechanisms of change, the question arises whether the results of process research on face-to-face therapy may be applicable to the online setting (Andersson \& Carlbring, 2008). Additionally, it is possible that important therapeutic processes have not been measured or are much too fine-grained or complex to be detected in existing studies.

Systematic case studies provide a particularly relevant method for shedding light on the processes of both face-to-face and online therapy, and may provide insight into how change happens or what obstacles patients could face that prevent change (Fishman, 2011, 2013). While 
effect sizes do not tell us about the different ways in which patients work with the treatment that leads to a therapeutic success or failure, and while inferential statistics fail to identify consistent predictors of outcome, systematic case studies are an "opportunity to investigate holistically, precisely, and in detail the individual and interactive roles of a variety of factors that affect the outcome for each specific client” (Fishman, 2011, p. 243). (For more on the advantages of studying contrasting-outcome cases drawn from the experimental condition of an RCT, see Fishman, Messer, Edwards, \& Dattilio, 2017). This approach is in line with the Evidence-Based Practice in Psychology model proposed by the American Psychological Association (2006), which views the integration of quantitative and qualitative results as the basis for an optimal therapy practice.

In the field of ICBT, case studies are still rare. Only recently, some case studies have started to focus on individuals’ progress in web-based treatments (Ooi, Raja, Sung, Fung, \& Koh, 2012; Patel, LaLima, \& Schmidt, 2015; Pugh, Hadjistavropoulos, Klein, \& Austin, 2014; Spence et al., 2008). Most of the current case studies focus on prototypical users in order to illustrate how ICBT is delivered in clinical practice. For example, Pugh, Hadjistavropoulos, Britt and Austin (2014) demonstrated in their case study on clinician-guided ICBT for depression how therapists can support patients working through a self-help program, and identified therapist statements that were perceived as helpful. As another example, a case report by Spence et al. (2008) highlighted the difficulties encountered while treating children with anxiety disorders online, such as monitoring issues, delays in session completion, or establishing an exposure hierarchy without the face-to-face aid of a therapist. Concerning SAD, case studies on ICBT are almost non-existent, even though they hold the promise of greatly increasing our understanding of the therapeutic process in SAD clients.

\section{Design of the Current Study}

In light of the above discussion, the current study describes and discusses two male clients with SAD who were drawn from the individual-guidance experimental condition of an RCT. The two clients had contrasting treatment outcomes. One, with a positive outcome, we call "Daydreamer.” The other, with a generally negative outcome, we call "Night Owl."

By applying the "Individual-Case-Comparison (ICC)" method proposed by Fishman (2008, 2011; Fishman et al., 2017), we attempt to extend the knowledge on quantitative group data from the RCT with qualitative case data from the case studies in order to better understand why one particular individual does or does not benefit from the intervention.

Another goal is to describe in clinical detail how guided self-help for SAD is implemented within the contextual complexities of an individual case, and to provide insight for clinicians interested in providing web-based treatments to particular clients. Thus we address opportunities and challenges in supporting clients while working through a web-based self-help program, including such issues as deadlines, progress monitoring, and adherence.

\section{Treatment Context}

The Daydreamer and the Night Owl described below received a clinician-guided, webbased CBT intervention for SAD in the context of the RCT study described above (Schulz et al. 
(2014, 2016). One of the primary goals of this RCT was to compare the efficacy of a novel webbased treatment format that included group-mediated peer support (building on the therapeutic principles of group therapy) with the more common format of ICBT, with individual therapist support. For the following case studies, we selected clients who received individual guidance, as this represents the most common setting for ICBT. We chose the two clients with contrasting outcomes who had some similarities in how they presented. Specifically, both were collegeeducated males with formally diagnosed SAD who had never (Daydreamer) or had not for 25 years (Night Owl) received psychotherapy for their disorder; and both were still able to function (Daydreamer as a college student, and Night Owl as an engineer).

\section{THE CLIENTS}

"Daydreamer," the client with the positive outcome, was a 23-year-old male geography student. He was single and lived in a shared apartment in a mid-sized city in Germany. In spite of his SAD symptoms, he had never been in psychological treatment before and was not on medication when he registered for the study.

"Night Owl," the client with a negative outcome, was a 41-year-old father of two children. Since the divorce from his wife two years prior to treatment, he had lived alone in a city close to his ex-wife and children. He worked as an engineer in a small company in Germany and had recently been promoted. He did not take any medication and reported having been in psychological treatment for SAD as a teenager.

\section{GUIDING CONCEPTION}

\section{Description of the Intervention and the Theoretical Model It Is Based Upon}

The main component of the treatment was a self-help guide for SAD adapted to the Internet. The 12-week treatment has been successfully evaluated in previous studies (Berger et al., 2011; Berger, Hohl, \& Caspar, 2009, 2010; Boettcher, Berger, \& Renneberg, 2012; Shulz et al., 2016). The program is based on the well-established cognitive-behavioral treatment model by Clark \& Wells (1995) and consists of eight text-based Modules, including several exercises (such as progressive muscle relaxation) and cognitive and behavioral worksheets (e.g., protocols for anxiety-inducing situations). Table 1 presents an overview of the content of the self-help program. Treatment sessions are made accessible in a sequential order, and each session takes about 60 minutes to complete. Participants are asked to complete one session per week, repeat the exercises and fill out the worksheets. While working with the program, clients are guided by a therapist (see below for details).

\section{Guidance and Communication}

As mentioned above, therapist guidance seems to be a way of increasing the efficacy and reducing the drop-out rates in self-help treatments. While working through the web-based material, the clients presented in this study were individually guided by a psychotherapist in her second year of CBT training in clinical psychology. Once a week, the therapist pro-actively contacted the clients via an integrated message function in the Internet-based program, and she 
provided feedback on the client's progress. In order to protect their privacy, clients chose a nickname at the beginning of treatment and were addressed by this pseudonym throughout. The clients could contact the therapist as often as desired, and would get a response within three working days. The therapist first initiated contact by introducing herself and outlining the procedure via a first therapist message. Participants were encouraged to ask questions and to report any problems they faced with the self-help program.

Since the main component of the intervention was the self-help material, therapist contact mainly focused on providing a structure and keeping up the participants' motivation to work on the self-help material. Also, the therapist gave feedback on individual entries concerning anxietyinducing situations, negative thoughts, exposures conducted, and/or on the explanatory model of their problems. In ICBT, the time it takes to provide feedback and respond to questions from clients can vary, ranging from only providing a brief encouragement to writing longer and more therapeutic responses to clients. However, on average it takes the therapist about 10 minutes per week per client.

\section{Rationale for Selecting Patients}

Our aim was to illustrate the different courses of treatment by selecting participants with a positive and a negative outcome who were drawn from our randomized control trial (Schulz et al., 2016; Fishman et al., 2017). Thus, we selected individuals according to (a) their treatment gains on primary social anxiety measures (Social Phobia Scale [SPS]) and Social Interaction Anxiety Scale, and to (b) their diagnostic status right after treatment. We first selected participants who had a positive outcome defined by a high combined-change score on the selfreport measures and who no longer met the diagnostic criteria for SAD at post-treatment. Accordingly, negative outcome cases were operationalized by a low or negative change score and by still meeting the SAD criteria after 12 weeks. From this pool of extremes, we let our clinical experience in ICBT guide us to select those individuals who provided what we believed were prototypical user patterns and who depicted a variety of client behaviors. Both clients' identities were disguised to protect their confidentiality.

There is one very important point when looking at cases with a poor outcome. We chose Night Owl as an example because he used the program thoroughly, established a stable relationship with his therapist, and still did not benefit sufficiently from the treatment. This pattern was representative for some clients who were still diagnosed with SAD at the end of the treatment. It should be noted, however, that there is another group of clients with a poor outcome in Internet-based guided self-help treatments: participants who stop using the treatment early and finally drop out. We encountered such cases as well, but we were often not able to contact them and reasons for treatment termination remained unclear. 


\section{ASSESSMENT OF THE CLIENT'S PROBLEMS, GOALS, STRENGTHS, AND HISTORY}

\section{Standardized Assessment Measures in the Randomized Control Measures That Were Employed In Assessing the Two Cases}

Daydreamer and Night Owl went through the assessment process employed with all the clients in the randomized controlled trial (RCT; Schulz et al., 2014, 2016). In the RCT, the initial study inclusion criteria were: (a) being at least 18 years old; (b) providing written consent; (c) having access to a computer with Internet connection; (d) having sufficient German language skills; (e) exceeding a pre-defined cut-off score on the social anxiety measures of the Social Phobia Scale (SPS) or Social Interaction Anxiety Scale (SIAS; see below for more on these two measures); (f) meeting the diagnostic criteria for SAD according to the diagnostic telephone interview; and (g) agreeing to undergo no other psychological treatment for the duration of the study. To determine the diagnostic status, psychologists used the Structured Clinical Interview for the DSM-IV for Axis I Disorders (SCID-I; see below). Participants with active suicidal plans, a history of psychotic or bipolar disorder, or unstable medication were excluded and referred to a local psychiatrist.

The primary and secondary symptom measures are listed below. All were collected at baseline, during therapy, and at follow up.

\section{$\underline{\text { Primary Outcome Measures }}$}

- $\quad$ The Social Phobia Scale (SPS) and the Social Interaction Anxiety Scale (Mattick \& Clark, 1998; Stangier, Heidenreich, Berardi, Golbs, \& Hoyer, 1999). Both are highly validated and utilized self-report measures of Social Anxiety Disorder. The SPS instrument is designed to assess anxiety in performance situations, such as public speaking or being the center of attention. The SIAS is designed to assess anxiety when in interacting with others in pairs or in groups.

- $\quad$ The Structured Clinical Interview for the DSM-IV for Axis I Disorders (SCID-I; First, Spitzer, Gibbon, \& Williams, 1995). This is a psychometrically established, semi-structured interview administered by a mental health professional to operationally define the presence of a variety of Axis I disorders in DSM-IV, including Social Anxiety Disorder (also called "Social Phobia").

\section{$\underline{\text { Secondary Outcome Measures }}$}

The secondary outcome measures included the following standardized, self-report measures:

- $\quad$ The Beck Depression Inventory (BDI-II; Hautzinger, Keller, \& Kühner, 2006), which assesses depressive symptoms, in the context of the high comorbidity of SAD and depression (Schulz et al., 2014). 
- The Brief Symptom Inventory (BSI; Franke, 2000), which measures a broad range of additional psychiatric symptoms, to further assess for comorbidity.

- The Inventory of Interpersonal Problems (IIP; Horowitz, Straub, \& Kordy, 2000), which identifies sources of relational distress and problems.

\section{Client Satisfaction}

- The Client Satisfaction Questionnaire (ZUF-8; Jürgen Schmidt, Lamprecht, \& Wittmann, 1989), which assesses global client satisfaction.

\section{$\underline{\text { Process Measures }}$}

- $\quad$ SPS and SIAS Over Time. Clients filled out the SPS and SIAS every two weeks to monitor treatment progress.

- The Working Alliance Inventory (WAI; Horvath and Greenberg, 1989) was administered to assess the patient's perceived quality of the therapeutic relationship. As in previous research on ICBT, we used a modified version of the WAI to account for the self-help character of the intervention (Berger et al., 2013). We were especially interested in the bond subscale, which measures the perceived empathetic bond between patient and therapist. Items such as "I think the psychologist likes me" and "The psychologist really cares about my well-being" are rated on a Likert scale ranging from 1 (“seldom”) to 5 (“always").

- $\quad$ Program usage. To learn more about the way users navigate through the program, we tracked the time spent in the program, the number of lessons completed, the number of diary entries, and the number of messages between clients and therapists. (For the results on program usage, see Section 7 below, Therapy Monitoring and Program Use.)

\section{Assessment of Daydreamer}

\section{Presenting Problems}

When asked about his main problem during the initial clinical interview, Daydreamer reported having low self-esteem caused by his fear of social situations, especially when talking to strangers on the phone or in other public settings. He usually prepared for a phone call by writing down exactly what he wanted to say and stated that when he picked up the receiver, he was so anxious that he could hardly speak. His fear of public speaking occasionally resulted in panic-like states. Small talk, or eating and drinking in a group, were also difficult activities for him. He usually tried to avoid such situations, which proved increasingly difficult during his studies. His fears included saying something wrong, misspeaking, and therefore being ridiculed and laughed at. In one-on-one situations, he felt that he was not able to contribute to the conversation. When in an anxiety-inducing situation, he experienced strong physical symptoms such as blushing, sweating, and shaky hands. 
When asked if he had experienced any other difficulties in the prior month, Daydreamer reported feelings of inadequacy, especially regarding his performance at his university, and decreased interest in his formerly beloved hobbies. He denied suicidal ideation by stating that he "could never do such a thing to his family" and that he was convinced life could be beautiful if he could overcome his problems.

\section{Problem History}

Daydreamer stated that he had always been a shy person, but his anxiety developed after being a victim of bullying in $7^{\text {th }}$ and $8^{\text {th }}$ grade. After he changed schools, the bullying stopped but the fears of being judged remained. He also reported having recurring episodes of depressive mood since the traumatic experiences at school. He had hoped that he would grow out of his social anxiety when he started going to college and moved to a new city. When the problems did not improve, he decided to look for help. He had been considering seeing a therapist and even searched for suitable options, but in the end he could not bring himself to finally make an appointment. He also worried about what people would think if they saw him enter a therapist's practice. Looking for alternatives, he found our study homepage via Google.

Diagnostic Status, and Primary and Secondary Outcome Measures At Baseline.

Prior to treatment, on the SCID-I Daydreamer fulfilled the DSM-IV criteria of SAD and of a Major Depressive Episode. This corresponded to the results on the self-report measures, as shown in Table 2. Specifically, the scores on the primary and secondary outcome measures were significantly elevated, above the clinical cut-off points.

\section{Assessment of Night Owl}

\section{Presenting Problems}

During the diagnostic interview conducted by telephone, Night Owl, a 41-year-old engineer, immediately stated that his main problem was his social anxiety. When asked to elaborate, he reported not being able to approach other people in informal situations. In situations of small talk with groups of people, he experienced blushing, red spots on his face and neck, as well as sensations of heat and strong sweating. He was convinced that it would be devastating for him personally and professionally if anyone noticed these physical symptoms because they clearly showed that "there was something wrong" with him. This was especially prevalent when talking to women he found attractive.

Interestingly, the problems did not occur when the interaction was strictly professional, as he stated that he could "hide behind the professional mask." Night Owl also worried a lot about the impact of his difficulties on his life, for example regarding his career or his wish to find a new partner. His worries caused him problems falling asleep at night; he would lie awake, replaying and dissecting conversations he had that day. 


\section{Problem History}

Night Owl stated that he was no stranger to feelings of social insecurity, which had started when he was a teenager. He could not name any specific events leading up to the development of his anxiety. Because his grades deteriorated and he withdrew more and more from both his classes and from others outside his family, his parents suggested he see a therapist.

Night Owl was in psychological treatment for SAD for one year at the age of sixteen. He stated that the therapy had helped him a lot with his fear of rejection at the time. Even though he remained introverted and rather insecure throughout the following years, the problems had only returned recently when he found himself in a new, challenging situation after the divorce from his wife. The couple had been arguing a lot about a variety of topics, mostly parenting and financial issues. His ex-wife then decided to file for divorce and Night Owl moved out of the family home. As Night Owl had recently been promoted at work and was confronted with his new situation as a single person, he felt that his old difficulties increasingly impaired his everyday functioning. He found our study homepage during one of his regular searches for information and novel treatment formats for SAD on the Internet.

\section{Diagnostic Status and Primary and Secondary Outcome Measures at Baseline}

On the SCID-I, prior to treatment, Daydreamer's symptoms met the DSM-IV criteria of SAD. This is reflected in Table 2 in his SPS score of 32 and his SIAS score of 58, both well above the clinical cut-off. According to the SCID-I, Night Owl had experienced a prior episode of major depression one and a half years ago after his divorce. At intake, he indicated no current depressive symptoms and denied suicidal ideation, reflected in his low BDI-II.

\section{CASE FORMULATION}

As planned, individual case formulations for Day Dreamer and Night Owl were not completed. Rather, designed into the Social Anxiety Disorder ICBT program is a generic case formulation for each client who uses the program. In addition, as described below, each client was guided by the ICBT program to develop his own personal case formulation.

\section{COURSE OF TREATMENT}

\section{Daydreamer's Course of Treatment}

\section{$\underline{\text { Week } 1}$}

In line with Module 1 (see Table 1 for a list of the modules), Daydreamer established reasons to initiate change and was asked to imagine what his life would be like if he overcame his fears. He stated that his SAD restricted him in private relationships and in professional settings; without his anxiety, he would be able to find friends to study and go out with. As a result, his grades would improve and he would be able to form deeper connections with people around him. Professionally, he realized that his anxiety kept him from applying to the internships in which he was very interested; instead, he was stuck with the little that he could get. As a more 
general reason for change, he found that his anxiety and constant worrying about being perceived as "weird" was taking up a huge part of his energy, energy that he would rather invest in hobbies or discovering his talents.

Daydreamer also had his first experiences with the long version of progressive muscle relaxation (PMR). During his first week, he practiced almost daily and consistently noted a marked change in his level of relaxation after the exercise: usually from 3 out of 10 prior to the exercise, with 10 being maximum relaxation, to 8 out of 10 afterwards. In the comment section in the program he wrote that he was able to shut out worries about his upcoming school test during the relaxation exercise. He usually practiced before going to bed and reported falling asleep more easily afterward.

In his online diary, Daydreamer tracked various difficult social situations. One example was his cousin's party invitation. As he related thoughts, he noted that he feared that he could not contribute to the conversation, that there would be too many strangers, and that everybody would reject him. His cousin would be ashamed of him and all the guests would wonder about the "weird cousin.” He rated his fear regarding this situation with a 6 on a scale of 1 to 10, with 10 being maximum fear. Physically, he felt constriction in his chest area and heart palpitations. In the column on behavior he stated that he would try to stay in a corner of the room and soon find an excuse to leave.

After the first week, the therapist gave feedback to reinforce the program use.

- “You’ve already completed the first lesson and started doing all of the exercises. Good Job!”

She highlighted the importance of individual goals to stay motivated:

- “Working on one's fears can be hard sometimes and it can be helpful to remind yourself of your goals. I understand that the goals you noted are important! Together, we will now set off on a journey towards reaching those goals one step at a time.” Job!”

"You’ve already completed the first lesson and started doing all of the exercises. Good

She highlighted the importance of individual goals to stay motivated:

- "Working on one's fears can be hard sometimes and it can be helpful to remind yourself of your goals. I understand that the goals you noted are important! Together, we will now set off on a journey towards reaching those goals one step at a time.”

The therapist also gave encouraging information about the next session to keep Daydreamer's interest high:

- 'In the next session you'll learn more about the development of social anxiety and its maintaining processes. I'm sure you'll find interesting suggestions there.”

In response, Daydreamer also wrote his first message to his therapist: 
- "Thank you very much for your kind feedback and thanks again for letting me use the program. To me, beginning this treatment was a great relief and beam of hope at the same time. I finally have the feeling that I can do something about the way I feel. When I read the material, I feel like every sentence is written about me personally!

At first, I was skeptical about the relaxation exercise. But when I tried it, I saw that I could just let my negative thoughts go and focus on the exercise. I felt so much more relaxed. Right now, I'm quite enthused about the treatment and looking forward to the next lesson. Kind regards, Daydreamer"

\section{Week 2}

Daydreamer's high motivation for change was also apparent in Module 2 (see Table 1), when he established an individual model for his difficulties. The amount of detail in his related notes was remarkable.

In line with Module 2's exploration of the development of the individual client's SADthat is, Daydreamer's own case formulation for his SAD-Daydreamer stated that he had always been a shy and introverted child who had to be prompted by his parents to speak to relatives. Daydreamer speculated that there might be a hereditary aspect to his anxiety since his mother was an introverted person herself. In his developmental history, he precisely recalled several hurtful incidences in his childhood and adolescence during which he had felt strongly humiliated by his peers. He stated that he had been repeatedly ridiculed because of his shyness, and he recalled at least one occasion when a teacher called him out in front of the whole class because he never contributed to the class discussion. As a result, he drew back even more and formed a self-concept of being socially inept and clumsy, thus avoiding even more situations-a vicious cycle he now understood was maintaining his anxiety. He concluded his model with: "Every time I want to try something, the thought 'you can't do that' comes up. I am my own worst enemy!”

In her weekly message, the therapist complimented the client on his very precise observations. It seemed important to shift the focus away from the past and toward the current behavioral and cognitive aspects:

- "You did a very good job with your personal anxiety model. I am sorry to hear that you experienced these hurtful things when you were younger. I can imagine how these things can make a lasting negative impression. Unfortunately, we cannot change what happened in the past. But, as you pointed out, there are several things you can do right now to reduce the stress and anxiety. One aspect would be working on the negative thoughts you have about yourself."

\section{$\underline{\text { Week } 3}$}

Daydreamer responded positively to the contents of Module 3 about cognitive restructuring. In a message to his therapist, Daydreamer stated that he was surprised to see how negatively he usually thought of himself and his behavior, which he had never noticed before: 
- 'It's like I’ve been always talking so negatively to myself in my mind. No wonder it made me feel bad!”

He used this new insight and the techniques he learned to work on his dysfunctional assumptions in a thought diary. As a first entry, he chose to examine the previously mentioned situation of his cousin's party. Table 3 shows an excerpt from Daydreamer's thought diary. He was invited to question how realistic his initial assumption was (from $0 \%$ to $100 \%$ true) and how fearful he was feeling (from 0 , least fearful, to 10, most fearful), and to try to find alternative explanations. Then, he noted what the consequences of his imagined horror scenario would be. Next, he re-evaluated the degree of the degree of truthfulness of his initial thought and the imagined consequences.

\section{$\underline{\text { Week } 4}$}

The next week, Daydreamer kept logging on regularly and completed all the exercises on attention training and detached mindfulness (see Module 4). He wrote to his therapist that he felt that those exercises were helpful, but experienced difficulties with the new, shorter relaxation exercise:

- "I find it difficult to concentrate on the new version. I usually fall asleep before I can complete the exercise.”

The therapist answered that this was something a lot of people experienced and suggested practicing in contexts other than before going to bed at night:

- "If you try practicing in different settings, for example while sitting in a chair in the kitchen or during a break at university, it will be easier to apply the techniques right before anxiety-inducing situations.”

\section{$\underline{\text { Week } 5}$}

Module 5 prepared Daydreamer for the confrontation with difficult situations in real life and established an individual exposure hierarchy. The psychologist encouraged him to take those next steps:

- "Dear Daydreamer,

You have now arrived at Module 5, which can be seen as the heart of the treatment. I understand that confronting your fears is challenging, but when I look at your progress so far, I am sure that you will succeed here as well. Please feel free to contact me if you have questions or are unsure about the next steps. Kind regards, ...”

Unfortunately and surprisingly to us, Daydreamer did not reply. We could see that he logged on, but he did not make any progress nor start with the protocol on testing reality in Module 5. On reflection, we realized that the exposure portion of the therapy is particularly challenging and so our initial surprise was tempered. After a week had passed, the therapist sent another message: 


\section{- “Dear Daydreamer}

It seems that you haven't found the time to continue working on the program. May I ask what makes you hesitate? I understand that this week's task seems tough so let me know if there is anything I can assist you with. I am looking forward to hearing from you. Kind regards, ...”

A few days later, Daydreamer responded:

- "Dear Therapist,

I'm sorry that I haven't continued with my work here. I feel really bad about it. Although I understand that confronting things is important, I just cannot see myself doing things I have avoided for so long. It all seems too much! I feel like all the courage I had has left me. While working on the program, I found out how much my social phobia has impacted my life. Now I'm scared and I'm afraid that I have wasted too much time doing nothing that I won't be able to catch up anymore. How much time do I have left in the program?”

The therapist felt this was a crucial moment in the therapeutic relationship. It seemed necessary to keep a positive outlook and to not let the client glide deeper into a spiral of selfdoubt and guilt.

- “Dear Daydreamer,

Thank you for your message and for sharing your concerns with me.

From your message, I understand that you are currently feeling a little overwhelmed and that you worry about your progress. So let's take a look at your concerns:

It's normal that it can be hard to work on one's fears and I also understand that it can be frightening to realize how much the anxiety affects one's life. A lot of our clients report similar feelings. As painful as this realization might be at first, it is also very important. We can only solve a problem if we recognize and name it - thus, you have already made important progress. There is obviously a lot you want to change and that's a good thing! Let's just start with small steps, for example with a difficult situation on the lower end of your anxiety hierarchy.

Concerning the duration of the program: We still have more than five weeks left to work together, so that's plenty of time. It would be great if you could start with the first confrontations soon if you feel ready for it, so I can support you. But even after 12 weeks, you can still work on the program independently, so there is no need to put yourself under pressure. Take one step at a time, everything else will come with time and practice.”

\section{$\underline{\text { Week } 6}$}

A couple of days passed until we heard back from Daydreamer, with the first entry in the protocol for testing reality and behavioral experiments (see Module 5). He reported that he tried something that "might seem small to others," but was the first successful experience for him. He noted in the diary that he had started to purchase his train tickets at the ticket counter instead of 
the vending machine. Although the interactions were short at first, Daydreamer felt that this was his first step of giving up his avoidance behavior.

In a related message he wrote:

- "I could have just gone to the vending machine like I always did, it would have been much easier for me. But I did what I was avoiding all along. It wasn’t as bad as I thought! It might sound stupid, but I was a little proud of myself afterwards.”

As this was regarded as crucial treatment progress, the therapist's next message aimed at reinforcing Daydreamer's courage and complimenting him on his breakthrough.

$\underline{\text { Weeks 7-12 }}$

During the following weeks, Daydreamer's exposure diary filled continuously with increasingly difficult exercises, which reflected his personal anxiety hierarchy. After his experiences with the train tickets, he started greeting his neighbors when he met them in the hallway and tried to contribute to class discussions at his university at least once every day.

In a message he stated that the information on a healthy lifestyle in Module 7 (see Table 1) inspired him to take up one of his old hobbies again and that he, therefore, signed up for a local volleyball team.

By Week 10, Daydreamer had completed all eight Modules, while regularly practicing relaxation techniques and trying out his new skills in real life, and continued these new skills through Week 12.

Shortly before treatment ended, the therapist received a message in which Daydreamer announced that he had an interview for an internship at a company he was particularly interested in and asked for advice. The therapist complimented him on his progress and together, they worked on a plan to prepare for his interview. In his last message, Daydreamer shared good news:

- 'I've landed an internship at a really prestigious company and I'm so happy about it! Thanks for your support!”

\section{Night Owl's Course of Treatment}

The therapist's first impression of Night Owl as a person who was ambivalently motivated to deal with his condition was confirmed from the beginning of his treatment. As reasons for initiating change, Night Owl stated that the biggest impairment was the fear itself. He felt strong anxiety in anticipation of social situations and in actual social interactions he found himself too tense to have an authentic exchange with others. He felt this was impairing his professional path, as well as his wish to find a new partner.

Another personal goal was to stand up for himself more often and to not let others take advantage of him. 
In the first exchange of messages, Night Owl accepted an offer mentioned in the material which involved asking the therapist to send him articles about web-based treatments of SAD. The therapist interpreted this behavior as further proof of Night Owl's need to establish a deeper understanding of his problems, but also as a sign of ambivalence about whether the treatment could be beneficial for him. The therapist agreed to send him the articles and encouraged Night Owl to contact her if he wanted to discuss the papers further.

\section{$\underline{\text { Week } 1}$}

Night Owl did not come back to the topic of efficacy-related material about web-based treatments, but rather he committed himself to the tasks of the first week (see Module 1in Table 1). He was responsive to the relaxation exercises in Module 1 and practiced almost every day. Without much prompting, he started to practice PMR before difficult situations, such as dates with women he met on an Internet dating site. His extensive identification and memory of anxiety-provoking situations in the corresponding protocol showed that his anxiety impaired more areas of his life than either he or his therapist expected. Other sources of distress were interactions with his ex-wife and doubts about his ability as a father.

\section{Week 2}

Night Owl perceived Module 2 on psychoeducation as a helpful overview even though the information presented was not entirely new to him. He wrote to his therapist,

- "It is very good to have all the information put together like this; it makes a lot of sense to me."

At the end of Module 2, in response to the development of his own case formulation for his SAD, Night Owl established an explanatory model for his problems, distinguishing between causal and maintaining factors. He described having an overprotective mother and a very critical father with high expectations. He reported being a sickly child, which seemed to reinforce his self-image of being weak. In his childhood, religious beliefs were used to create an atmosphere of pressure and punishment, since he was constantly told that "only well-behaved children with good grades go to heaven.”

In these surroundings, he described suffering from a constant fear of rejection from an early age. Regarding factors that maintained his anxiety in the present, he indicated that he avoided situations since he felt "there was no use in trying." He explained that no matter how positive the experience was, he always found something to criticize about himself. He also felt constantly physically and mentally stressed because he was either dissecting past situations or worrying about future interactions. He realized that stress kept him from acting naturally in social situations. The therapist invited him to use these insights in Module 3, on cognitive restructuring.

\section{$\underline{\text { Week } 3}$}

Night Owl used the cognitive restructuring techniques introduced in Module 3 to work on a variety of dysfunctional assumptions, beyond those related to social anxiety. He seemed to 
realize that his negative thinking patterns and worrying were impacting his daily functioning also outside of social interactions as well (for an example see Table 4).

The therapist encouraged him to use the cognitive restructuring protocols whenever he felt it would be helpful:

- $\quad$ "Dear Night Owl,

I was happy to see how much you're using the protocol for realistic thinking. For instance, I found the way you worked on the playground situation to be very impressive. You questioned the basis for the assumption ("Who are those people that I think are judging me?"), discovered that the relationship to your daughter was not in danger, and came to the conclusion that there is no realistic reason to blame yourself.

Maybe you also found that negative thoughts appear almost automatically and usually can't withstand critical scrutiny. Keep up the good work! It would be great if you could continue working on your negative beliefs in just the same way. Kind regards, ..." reply:

Despite this encouragement, it seemed that Night Owl's skeptical side prevailed in his

- "Dear Therapist,

Thanks a lot for the personal and encouraging feedback.

I have a question: I've worked on up to two situations per day and always came to realize that the thoughts were unrealistic. Despite this insight, I don't see any changes: The next day, I still think about it the exact same, negative way. Would you recommend working on more situations? Or is it enough and just takes time? Kind regards, Night Owl."

In this message Night Owl showed further signs of doubt and gave the impression that his difficulties were not improving as quickly as he wished. This seemed consistent with his assumed pattern of perfectionism and putting himself under a great deal of pressure. It seemed crucial to take his concerns seriously, and at the same time encourage him to be patient with himself.

- "Dear Night Owl,

Thank you very much for sharing your concerns with me. I see that you have kept on working regularly and that's great. But I also sense that you might feel it's not going fast enough.

The insight that your negative assumptions are not realistic is a first important step. And it is just like you assumed: It takes a lot of practice, time and work on more situations. Please remember how many years you employed this negative way of thinking and how well "trained" this line of thinking is. It's not surprising that it will take some time and practice to un-learn it and practice a new perspective. Just try to give it more time; you're moving in the right direction. Kind regards"

Night Owl thanked his therapist for the feedback, which he considered to be "informative and motivating." 


\section{$\underline{\text { Week } 4}$}

During this week, Night Owl indicated that he had positive experiences with the mindfulness exercises and used them to deal with his worrying, especially before going to bed. Also, he seemed responsive to the idea of actively shifting his attention to external stimuli. "I'm usually so fixated on my thoughts and how others might perceive my behavior that I don't even notice what's going on around me! I should really try to concentrate on the outside in the future," he wrote to this therapist.

\section{$\underline{\text { Week } 5}$}

When it was time for the in vivo exposure in Module 5, Night Owl carefully created an exposure hierarchy, starting with small talk situations in the office kitchen and working his way up to the most difficult situations, such as openly disagreeing with his ex-wife and contacting women in whom he was interested. When asked how he felt about starting the exposure, he told his therapist that he wanted to take a short break before putting his plans into action, knowing things were "about to get serious." He seemed confident that he would be able to face an anxietyprovoking situation, but expressed worries that he would take the "easy way out," as he described himself as a "master of muddling through."

As an example, he analyzed what happened when he was out on a date. The atmosphere was usually pleasant, but he did not allow himself to feel genuine closeness or any emotional attachment because he was afraid of rejection. As a consequence, he came up with reasons as to why he was not that interested in the woman after all. The therapist congratulated the client on his ability to reflect on his actions and explained the situation in terms of avoidance behavior. She encouraged him to apply the learned techniques of cognitive restructuring in a dating situation.

\section{$\underline{\text { Week } 6}$}

During this week, Night Owl took a break from the ICBT program.

\section{$\underline{\text { Week } 7}$}

Night Owl confronted a lot of different situations from his exposure hierarchy after his short break from the program in Week 6. He actively put himself in situations he would have usually avoided, and derived a heightened sense of self-efficacy from these experiences.

\section{$\underline{\text { Week } 8}$}

In week eight, Night Owl wrote to the therapist:

- "I really appreciate your feedback, especially when you focus on individual situations. I am proud to say that I can see first improvements! Even though I don't think my overall anxiety has decreased that much, I do more things that are interesting and fun now, which is an improvement of my quality of life.” 
On the other hand, Night Owl kept noting different kinds of what were in effect covert avoidance or safety behavior strategies. For example, he prepared telephone conversations by practicing what he wanted to say in his head and opening the windows to let fresh air in to combat his anxiety. It seemed important to the therapist to validate Night Owl's efforts, while still encouraging him to reduce his safety behaviors.

In accordance with Night Owl's expressed wish, the therapist pursued this goal by focusing on concrete situations and by positively highlighting the client's efforts to reduce the time spent to prepare for a social situation. For example, the therapist responded to a protocol entry about a phone call with a female acquaintance of the client.

- "You were able to call her without re-reading your last message exchange. And as you found out, it was a pleasant talk and you did feel confident. This must have been a very positive experience.”

\section{$\underline{\text { Week } 9}$}

By this week, the client's activity in the program seemed to be reduced. The following excerpt of a message shows that the client reached Module 7 at a very stressful period of his life:

- "There is just so much going on in my private and professional life, and I don't find the time to work on the protocols that much. The good thing is that there are so many situations that occur spontaneously and I get to practice without planning it. Right now, I am trying to follow the advice from Module 7 to control my high stress levels. I use the relaxation techniques and try to sleep and exercise enough.

The advantage of my busy schedule is that I simply don't have the time to over-analyze every message I sent or conversation I had. I will continue to fill out the diaries as soon as things quiet down a little bit."

\section{$\underline{\text { Weeks 10-12 }}$}

Keeping his promise, Night Owl continued to record his activities in the protocol on reality-testing throughout weeks 10-12, and showed a continuous decrease of experienced anxiety, even though he did not succeed in completely giving up his safety behaviors. He still prepared mentally for some of the difficult situations, but was able to reduce the time he needed to get ready for those situations.

The therapeutic communication during those last three weeks focused on individual situations Night Owl had confronted and the change of dysfunctional cognitions towards himself. In week 12, Night Owl reported that the time he spent worrying and being anxious before a situation had reduced, and that he started to experience moments of calmness, which he associated with the mindfulness-based exercises. 
At the end of treatment, Night Owl summarized his experience as follows:

- "I want to thank you for supporting me and including me in the study. Knowing that somebody was there to monitor my progress and to tell me that I was moving in the right direction was very helpful for me.

- On the one hand, I see a very positive development. My quality of life has improved a lot! I've become more active and I've tried new things. I feel that I've become a little more relaxed and more focused on the present. On top of that, I sometimes manage to stop worrying and over-analyzing.

- On the other hand, I've realized that I still act over-controlled and too formal in a lot of social situations. Even though I do not feel fear, per se, I am still very tense, and I think I don't appear, especially when I meet women. I hope this will get better with time and practice.

- In sum, I'm happy that I participated in the study and I want to continue practicing what I've learned."

In her last message, the therapist herself summed up Night Owl's progress and focused on the many positive aspects of what he wrote, including his important learning experiences. As for the last part of Night Owl's message, she discussed his observations in the context of safety behaviors.

- "You have learned a lot, confronted many situations, and noticed positive changes in your life. You state that there are still some situations in which you don't act as relaxed as you want to be. This is important, because it shows the way you may want to go in the future. I assume that acting 'overcontrolled' and 'too formal,' as you put it, might be part of a safety strategy of yours and it could be your next goal to slowly let go of this. But for now, you can be very proud of what you've accomplished in such a short time.”

\section{THERAPY MONITORING AND PROGRAM USE}

\section{Daydreamer}

Daydreamer completed all eight Modules of the program and spent a total of 36 hours logged on. Over 12 weeks, he sent 6 messages and received 14 from his therapist. In the different diaries, he made 35 entries (11 on relaxation, 14 in the protocol for anxiety-provoking situations ,and 10 in the dysfunctional thought diary). He spent 6 hours in the contact section of the program, whereas the therapist spent approximately 4 hours monitoring the client's progress and giving feedback. More details about these statistics are presented in Table 5.

\section{Night Owl}

Night Owl spent 34 hours logged on to complete the program. He composed 39 entries in the relaxation diary, recorded 57 anxiety-provoking situations, and worked on 33 dysfunctional thoughts. There were 39 entries in the protocol for testing reality. Also, Night Owl spent four 
hours in the contact section to send 11 messages, whereas the therapist spent 3 hours composing 17 messages and monitoring the client's progress.

\section{CONCLUDING EVALUATION OF THE THERAPY'S PROCESS AND OUTCOME}

\section{Post-Treatment: Daydreamer's Outcome}

\section{Daydreamer's Primary and Secondary Outcome Measures}

Table 2 shows that Daydreamers' self-report questionnaires after 12 weeks of treatment indicate significant reductions concerning the primary and secondary outcome measures. Regarding social phobia and depression, the scores at post-treatment were well below the cut-off and thus within the range of a healthy, nonclinical population.

\section{Daydreamer’s Diagnostic Status}

In the telephone interview after 12 weeks, Daydreamer did not meet the criteria for SAD anymore, nor did he report any depressive symptoms. Concerning his social insecurities, he stated that he still had a "queasy" feeling right before giving a presentation at his university, but he did not panic or have blackouts any more. He felt relieved that he now had "tools" to deal with feelings of anxiety and was happy to be able to do things he had avoided for a long time, such as joining a sports team and actively participating in class. For the future, he planned to keep confronting himself with difficult situations and, if necessary, to look at the material from the treatment again, which he had saved and printed.

\section{Daydreamer's Process Measures}

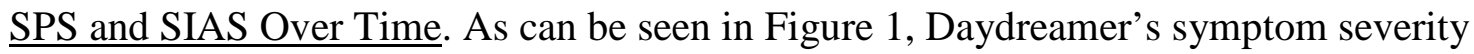
decreased significantly during the 12 weeks of treatment. The first two weeks demonstrated the steepest decline of symptom severity, which can be linked to the client's reported feelings of relief upon realizing that something could be done immediately after starting the treatment (see Daydreamer's first message to the therapist, quoted above). Another marked change in trajectory happened in the eighth week when starting the in vivo exposure. At post-treatment the SPS and SIAS measures of SAD severity were 8 and 28, respectively (see Table 2), below the predefined clinical cut-offs of 22 and 33, respectively.

Working Alliance Inventory (WAI). At the first administration of the WAI after week two, Daydreamer started with a score of 3.25 out of 5. The score slowly but continuously increased during the twelve weeks of treatment and the client terminated treatment with a score of 4.5 (which means that he was content with the therapeutic alliance most of the time).

\section{Daydreamer's Client Satisfaction (ZUF-8)}

Daydreamer's score was at 3.88 out of 4 on the Client Satisfaction Questionnaire (CSQ-8; Attkisson and Zwick (1982), stating that he was "very content” with the treatment he had 
received, that it "helped a lot" and "met most of [his] needs.” In the personal comments he noted that the therapist's guidance was crucial to him, especially by not putting him under pressure or reprimanding him when he had failed to complete a task. In his opinion, the parts about realistic thinking, relaxation and self-focused attention were the most helpful.

\section{Six-Month Follow-Up: Daydreamer's Outcome}

The diagnostic interview at follow-up revealed that treatment gains were maintained at the six-month follow-up. On the phone, Daydreamer told the assessor that he occasionally looked at his printouts of the clinical material from the program and that the questioning of negative thoughts had become integrated into his everyday routine. He reported feeling freer to do things he liked and had recently joined a theater group for introverted people. Regarding his attitude towards his problems, he had accepted that anxiety was at some times a larger, and at some times a smaller part of his life, but it no longer determined his behavior.

\section{Post-Treatment: Night Owl's Outcome}

\section{$\underline{\text { Night Owl’s Primary and Secondary Outcome Measures }}$}

Immediately after the treatment, social phobia measurements demonstrated a slightly decreased symptom severity in comparison to the baseline (see Table 3). However, the scores on SPS and SIAS were still within the range of a social phobic population. The BDI-II showed a substantial reduction, which corresponded with the self-reported increased social activity and perceived higher quality of life. This was also reflected in a lowered BSI score. However, there was no significant change in interpersonal problems as indicated by the IIP.

\section{Night Owl’s Diagnostic Status}

In the second diagnostic interview, Night Owl reported that his anxiety was clearly reduced but he still felt impaired by his symptoms. There were still some situations he avoided or only endured under distress, such as certain telephone calls or small talk situations with new clients at work. On the other hand, he stated that the overall impairment and time spent worrying before difficult situations were reduced. His most important learning experience was that confrontation was "not as horrible" as he had previously thought. Overall, the assessor concluded the client still met the DSM-IV criteria for SAD.

\section{$\underline{\text { Night Owl's Process Measures }}$}

SPS and SIAS Over Time. Night Owl's profile of symptom severity over the course of treatment is shown in Figure 2. The trajectory appears to reflect the individual gains and setbacks Night Owl reported during treatment. There is a marked decrease in week six, when he started the in vivo exposure and a peak during the period when he indicated being too stressed to work on the program. Overall, there is a tendency towards symptom reduction, even though at posttreatment the endpoints on the SPS and SIAS of 23 and 38, respectively (see Table 3), are still above the predefined cut-offs of 22 and 33 for these measures, respectively. 
Working Alliance Inventory (WAI). After the second week, the client indicated a high rating of the therapeutic alliance ( 4.0 out of 5 on the WAI). This score increased continuously until the last week of treatment, which ended with a score of out of 4.75 out of 5, which means that the client was "almost always" very content with the therapeutic relationship.

\section{Night Owl's Client Satisfaction (ZUF-8)}

Night Owl reported being “very content” with the treatment he had received (3.63 out of 4 on the CSQ-8). He very much liked the structured information provided by the treatment, the flexibility of use, and the possibility to monitor his own progress. When asked what he felt was missing, he indicated that he would have needed more support, such as in the form of synchronous communication via telephone or scheduled video conferencing with the therapist. He would also have appreciated stricter deadlines for assignments. In addition, he indicated that 12 weeks were not enough time to confront every relevant situation.

\section{Six-Month Follow-Up: Night Owl's Outcome}

As shown in Table 2, compared with baseline, there was a clear decrease in the Social Phobia score at follow-up. In contrast, the Social Anxiety Interaction Scale and the Brief Symptom Inventory were nearly as high at follow-up as at baseline. Additionally, at follow-up Night Owl demonstrated elevated levels of depression on the BDI-II in comparison to posttreatment. In the telephone interview, Night Owl reported that anxiety was still relevant to his everyday functioning, even though he did not avoid as many situations as before the treatment. According to the structured clinical interview (SCID-II), the criteria for SAD were still fulfilled.

Night owl still appeared to be highly motivated and active in working on his goals. When asked how he currently handled his problems, he stated that he continued using techniques learned during treatment, such as keeping a thought diary, writing down positive experiences, and employing mindfulness-based exercises. He also took to literature to find out more about the nature of his problems and was under the impression he had some avoidant-personality tendencies. He had recently decided to seek a face-to-face, cognitive-behavioral treatment in order to discuss this idea and to receive support for his exposure exercises.

\section{Cross-Case Comparison}

We selected these clients to illustrate different pathways and outcomes in a therapistguided self-help intervention for SAD. There are certain aspects we found to be crucial in order to understand the mechanisms of therapeutic change in this specific setting.

First, these cases illustrated certain shared characteristics. Both clients were highly educated, capable of introspection, and motivated to take part in the study. These intellectual abilities and motivational prerequisites might be especially beneficial for this type of intervention, since the amount of therapeutic support is limited. Also, their high ratings on the WAI suggest that the clients were able to form a stable relationship with the therapist despite the fact that the therapeutic contact was conducted exclusively online. In both cases, the clients were able to address difficulties in the written communication and stated that they found the 
therapeutic guidance to be crucial to their progress. The clients’ positive evaluation of the therapeutic alliance in the WAI is a common finding in research on Internet-based interventions, finding ratings roughly equivalent to those found in face-to-face therapy (Berger, in press).

An intriguing finding was that in these two cases, the better outcome was associated with a slightly worse rating of therapeutic alliance. This raises the question as to whether therapeutic alliance has a significant influence on treatment outcome in ICBT. Quantitative research on this issue reveals mixed results. Even though correlations between alliance and outcome are usually positive, they often fail to reach statistical significance. Thus, it has been suggested that in ICBT, in comparison to face-to-face therapy, the agreement on goals and tasks of the self-help material might be more important than the affective bond between client and therapist (Berger, in press).

One possible explanation for the mixed results is that for some clients, the self-help guide is sufficient to alleviate their problems and the therapeutic guidance is only necessary to keep their independent work "on track" and maintain their motivation. It can be assumed that in such cases, the therapeutic alliance does not have a significant influence on the treatment outcome. This observation is in line with our experience with guided self-help treatments and with a previous study in which one group of participants could choose how much therapeutic support they received (Berger et al., 2011). Only half of the clients asked for regular contact, leading to the conclusion that for some clients, support and the alliance was not as important as for others.

There is another observation that supports the notion that the importance of therapeutic alliance varies inter-individually. The two clients presented in these case studies not only showed different ratings of therapeutic alliance, but they also differed in the amount and quality of support they needed while working through the self-help program. While Daydreamer was content with the weekly and short, motivational or instructional messages, Night Owl required more tightly knit guidance. We saw that Night Owl presented his need for support by addressing more personal matters in his messages to the therapist and broaching topics that went beyond the scope of the self-help material, such as his assertiveness in everyday situations and difficulties in romantic relationships. It can be speculated that for clients like Night Owl, the client's selfdisclosure contributed to a better therapeutic alliance, which was necessary for their personal treatment gain. We tried to fulfill these different needs for support by tailoring the length and content of the therapist messages as much as the format and issues of comparability within the randomized controlled trial permitted.

The pattern we saw with Night Owl—a patient who benefitted some from guided ICBT, but who, by his own judgment, required further assistance in form of face-to-face therapy-was not uncommon, especially with clients who had no previous treatment history. To them, the selfhelp program was a low-threshold way to learn about psychotherapy. For some, the registration for the study was the first contact with mental health care. It can be assumed that if this first contact with a psychological treatment and a therapist is perceived as positive, it becomes easier for those clients to seek a traditional treatment afterwards.

As for the therapeutic guidance, there appears to be a thin line between contacting the client regularly to keep him or her motivated and applying too much pressure and, therefore, risking the client withdrawing. We learned from Daydreamer that he appreciated the therapist's 
non-judgmental reaction when he failed to complete an assignment, whereas Night Owl reported that he would have benefitted from a stricter deadline policy. Keeping this delicate balance is a therapeutic challenge, especially with the asynchronous, text-based communication and its lack of paraverbal and nonverbal cues. This dilemma became particularly apparent when Night Owl failed to log on for several weeks. We found that the most beneficial approach was to convey two messages. On the one hand, the therapist let Night Owl know that it would be a loss if he did not continue to build on their accomplishments. On the other hand, the therapist stressed that she would be available whenever he felt ready to continue. Asking if there was anything in particular that he was unsure about also proved to be an opportunity to engage Night Owl in an email exchange and in treatment.

Regarding the course of treatment, Modules 5 and 6 (see Table 1), typically planned in the fifth and sixth weeks, seemed to be a crucial moment. This may not come as a surprise since Module 5 contains the rationale for in vivo exposure, and Module 6 encourages clients to implement their own behavioral experiments. Confronting difficult, previously avoided situations is a core component in CBT for the treatment of SAD. Obviously, the idea of confronting one's fears is frightening for many clients (see for example the peak of Night Owl's symptom severity in week six). A lot of clients reported that these modules were the most challenging, but also the most helpful modules of the intervention. We used this experience and passed it on to other participants in order to encourage them and to increase adherence.

It also appears that there were certain individually relevant problem areas for Night Owl that were not targeted in the program, e.g., modules for assertiveness training. We did not screen for personality disorders and thus can neither confirm nor reject the clients' own tentative diagnosis of an avoidant tendency, but it can be speculated that such aspects influenced the treatment process.

Another characteristic feature of web-based interventions is the great flexibility of use. Clients are free to work on the program whenever and wherever they like. This is especially convenient for people with a busy schedule or those who are initially too impaired by their symptoms to seek face-to-face treatment (it was not uncommon for individuals interested in participating in the study to hesitate before agreeing to talk to the assessors over the telephone).

Interestingly, both Daydreamer and Night Owl indicated being similarly content with the treatment they had received despite the different treatment outcomes. It seems likely that even if Night Owl could not be considered recovered after 12 weeks of treatment, he still benefitted to some extent and was later able to use what he had learned to handle his anxiety.

As we noted above in the section on the "Rationale for Selecting Patients," Night Owl was chosen as a client with poor outcome because, in spite of his using the program thoroughly and establishing a solid relationship with his therapist, he still did not benefit from treatment in terms of recovering from his SAD disorder, as did Daydreamer. There are other types of pooroutcome clients in ICBT who stop using the treatment early and prematurely drop out. Systematic case studies of these clients would importantly complement the knowledge that comes from considering clients like Night Owl. 


\section{Conclusion}

The cases of Daydreamer and Night Owl demonstrate some of the opportunities and limits of ICBT for SAD. Daydreamer is a client who improved enough to be considered cured after the treatment with comparatively little therapeutic support; and Night Owl is a client who got better in some ways, but for whom face-to-face contact certainly seemed indispensable to consolidate therapeutic progress.

The cases of Daydreamer and Night Owl also show that it was possible to establish a stable working alliance and therapeutic relationship in a web-based intervention. Even though both patients appreciated the therapeutic support, in general the impact of alliance on treatment outcome may vary based on a client's individual needs and the therapeutic support should be tailored accordingly. From a practitioner's point of view, there are certain critical moments in the course of treatment when the therapist's encouragement and support are especially necessary to help the clients create change (for example, after Week 5 with Daydreamer, when he was starting with in vivo exposure). Special attention should be paid to keeping the balance between encouragement and monitoring of task completion.

As this in-depth analysis of two representative cases shows, ICBT for SAD clients is a promising approach that can result in significant symptom reduction, even though for some, the personal contact to a therapist seems irreplaceable.

\section{REFERENCES}

American Psychological Association. (2006). Evidence-based pratcice in psychology. American Psychologist, 61, 271-285.

Andersson, G., \& Carlbring, P. (2008). Predicting treatment outcome in internet versus face to face treatment of panic disorder. Computers in Human Behavior, 24, 1790-1801. doi: 10.1016/j.chb.2008.02.003

Andersson, G., Carlbring, P., Berger, T., Almlöv, J., \& Cuijpers, P. (2009). What makes Internet Therapy Work? Cognitive Behaviour Therapy, 38(1), 55-60. doi: 10.1080/16506070902916400

Andersson, G., Cuijpers, P., Carlbring, P., Riper, H., \& Hedman, E. (2014). Guided Internetbased vs. face-to-face cognitive behavior therapy for psychiatric and somatic disorders: a systemativ review and meta-analysis. World Psychiatry, 13, 288-295.

Andrews, G., Cuijpers, P., Craske, M. G., P., M., \& Titov, N. (2010). Computer therapy for the anxiety and depressive disorders is effective, acceptable and practical health care: A meta-analysis. PLoS ONE, 5(10).

Andrews, G., Davies, M., \& Titov, N. (2011). Effectiveness randomized controlled trial of face to face versus Internet cognitive behaviour therapy for social phobia. Australian and New Zealand Journal of Psychiatry, 45(4), 337-340.

Attkisson, C. C., \& Zwick, R. (1982). The client satisfaction questionnaire. Psychometric properties and correlations with service utilization and psychotherapy outcome. Evaluation and Program Planning, 5, 233-237. doi: 10.1016/0149-7189(82)90074-X 
Barak, A., Hen, L., Boniel-Nissim, M., \& Shapira, N. a. (2008). A Comprehensive Review and a Meta-Analysis of the Effectiveness of Internet-Based Psychotherapeutic Interventions. Journal of Technology in Human Services, 26(2/4), 109-160.

Berger, T. (in press). The therapeutic alliance in internet interventions: A narrative review and suggestions for future research. Psychotherapy Research.

Berger, T., \& Andersson, G. (2009). Internetbasierte Psychotherapien: Besonderheiten und empirische Evidenz. Psychother Psych Med, 59, 159-170. doi: 10.1055/s-0028-1090162

Berger, T., Boettcher, J., \& Caspar, F. (2014). Internet-based guided self-help for several anxiety disorders: A randomized controlled trial comparing a tailored with a standardized disorder-specific approach. Psychotherapy, 5, 207-219. http://dx.doi.org/10.1037/a0032527

Berger, T., Caspar, F., Richardson, R., Kneubühler, B., Sutter, D., \& Andersson, G. (2011). Internet-based treatment of social phobia: A randomized controlled trial comparing unguided with two types of guided self-help. Behaviour Research and Therapy, 49, 158169. doi: doi:10.1016/j.brat.2010.12.007

Berger, T., Hohl, E., \& Caspar, F. (2009). Internet-Based Treatment for Social Phobia: A Randomized Controlled Trial. Journal of Clinical Psychology, 65(10), 1021-1035. doi: 10.1002/jclp.20603

Berger, T., Hohl, E., \& Caspar, F. (2010). Internetbasierte Therapie der sozialen Phobie: Ergebnisse einer 6-Monate-Katamnese. Zeitschrift für Klinische Psychologie und Psychotherapie, 39(4), 217-221. doi: 10.1026/1616-3443/a000050

Boettcher, J., Berger, T., \& Renneberg, B. (2012). Does a pre-treatment diagnostic interview affect the outcome of internet-based self-help for social anxiety disorder? A randomized controlled trial. Behavioural and Cognitive Psychotherapy, 40(5), 513-528. doi: 10.1017/S1352465812000501

Boettcher, J., Carlbring, P., Renneberg, B., \& Berger, T. (2013). Internet-Based Interventions for Social Anxiety Disorder - an Overview. Verhaltenstherapie, 23, 160-168. doi: $10.1159 / 000354747$

Boettcher, J., Renneberg, B., \& Berger, T. (2013). Patient expectations in internet-based selfhelp for social anxiety. Cognitive Behaviour Therapy, 42(3), 203-214.

Botella, C., Gallego, M. J., Garcia-Palacios, A., Guillen, V., Banos, R. M., Quero, S., \& Alcaniz, M. (2010). An Internet-Based Self-Help Treatment for Fear of Public Speaking: A Controlled Trial. Cyberpsychology, Behavior and Social Networking, 23(4). doi: 10.1089/cyber.2009.0224

Chartier, M. J., Hazen, A. L., \& Stein, M. B. (1998). Lifetime Patterns of Social Phobia: A Retrospective Study of the Course of Social Phobia in a nonclinical Population. Depression and Anxiety, 7, 113-121.

Clarke, D. M., \& Wells, A. (1995). A cognitive model of social phobia. New York: Guilford Press.

First, M. B., Spitzer, R. L., Gibbon, M., \& Williams, J. B. W. (1995). Structured clinical interview for DSM-IV Axis I disorders (SCID-I). Washington, DC: American Psychiatric Press.

Fishman, D. B. (2008). Case studies of good and poor outcome in RCT clients: A new, "Individual-Case-Comparison" method for psychotherapy research. Paper presented at the Society of Psychotherapy Research, Barcelona, Spain. 
Fishman, D. B. (2011). The "Individual-Case-Comparison" Method for systematically comparing good-outcome and poor-outcome rct clients: Editor's introduction. Pragmatic Case Studies in Psychotherapy, 7(2), Article 1, 242-245. Available: http://pcsp.libraries.rutgers.edu

Fishman, D. B. (2013). The pragmatic case study method for creating rigorous and systematic, practiioner-friendly research. Pragmatic Case Studies in Psychotherapy, 9(4), Article 2, 403-425. Available: http://pcsp.libraries.rutgers.edu

Fishman, D.B., Messer, S.B., Edwards, D.J.A., \& Dattilio, F.M. (2017). Case studies within psychotherapy trials: Integrating qualitatve and quantitative methods. New York: Oxford.

Franke, G. H. (2000). BSI, Brief Symptom Inventory von L. R. Derogatis (Kurzform der SCL-90R) - deutsches Manual. Göttingen: Beltz Test GmbH.

Gandek, B., Ware, J. E., Aaronson, N. K., Apolone, G., Bjorner, J. B., Frazier, J. E., ... Sullivan, M. (1998). Cross-Validation of Item Selection and Scoring for the SF-12 Health Survey in Nine Countries: Results from the IQOLA Project. Journal of Clinical Epidemiology, 51(11), 1171-1178.

Hautzinger, M., Keller, F., \& Kühner, C. (2006). BDI-II Beck Depressions-Inventar. Frankfurt am Main: Harcourt Test Services.

Hedman, E., Andersson, G., Ljotsson, B., Andersson, E., Rück, C., Mörtberg, E., \& Lindefors, N. (2011). Internet-Based cognitive behavior therapy vs. cognitive behavioral group therapy for social anxiety disorder: A randomized controlled non-inferiority trial. PLoS ONE, 6(3), e18001. doi: 10.1371/journal.pone.0018001

Hedman, E., Furmark, T., Carlbring, P., Ljotsson, B., Rück, C., Lindefors, N., \& Andersson, G. (2011). A 5-year follow-up of internet-based cognitive behavior therapy for social anxiety disorder. J Med Internet Res, 13(2), e39. doi: 10.2196/jmir.1776

Hedman, E., Ljotsson, B., Rück, C., Bergström, J., Jansson, L., Andersson, E., . . Lindefors, N. (2013). Effectiveness of Internet-based cognitive behavior therapy for panic disorder in routine psychiatric care. Acta Psychiatr Scand, 128, 457-467. doi: 10.1111/acps.12079

Horowitz, L. M., Strauß, B., \& Kordy, H. (2000). Inventar zur Erfassung interpersonaler Probleme (IIP-D). Weinheim: Beltz.

Horvath, A. O., \& Greenberg, L. S. (1989). Development and validation ot the working alliance inventory. Journal of Counseling Psychology, 36, 223-233.

Jacobson, N. S., \& Truax, P. (1991). Clinical significance: A statistical approach to defining meaningful chance in psychotherapy research. Journal of Consulting and Clinical Psychology, 59(1), 12-19.

Jürgen Schmidt, Lamprecht, F., \& Wittmann, W. W. (1989). Zufriedenheit mit der stationären Versorung. Entwicklung eines Fragebogens und erste Validitätsuntersuchungen. Psychother med Psychol, 39, 248-255.

Kessler, R.C. (2003). The impairments caused by social phobia in the general population: Implications for intervention. Acta Psychiatr Scand, 108, 19-27.

Klein, J. P., Berger, T., Schröder, J., Späth, C., Meyer, B., Caspar, F., . . Moritz, S. (2013). The EVIDENT-trial: protocol and rationale of a multicenter randomized controlled trial testing the effectiveness of an online-based psychological intervention. BMC Psychiatry, 13(239). doi: 10.1186/1471-244X-13-239 
Ooi, Y. P., Raja, M., Sung, S. C., Fung, D. S. S., \& Koh, J. B. K. (2012). Application of a webbased cognitive-behavioral therapy programme for the treatment of selective mutism in Singapore: A case series study. Singapore Med J, 53(7), 446-450.

Palmqvist, B., Carlbring, P., \& Andersson, G. (2007). Internet-delivered treatments with or without therapist input: Does the therapist factor have implications for efficacy and cost? Expert Review of Pharmacoeconomics and Outcomes Research, 7, 291-297.

Patel, S. R., LaLima, C., \& Schmidt, A. B. (2015). Implementing Internet-based cognitive behavioral therapy for obsessive compulsive disorder: A case report. Annals of Psychiatry and Mental Health, 3(3).

Pugh, N. E., Hadjistavropoulos, H. D., Klein, B., \& Austin, D. W. (2014). A case study illustrating therapist-assisted internet cognitive behavior therapy for depression. Cognitive and Behavioral Practice, 21(1), 64-77. doi: 10.1016/j.cbpra.2013.08.002

Schulz, A., Stolz, T., \& Berger, T. (2014). Internet-based individually versus group guided selfhelp treatment for social anxiety disorder: Protocol of an randomized controlled trial. BMC Psychiatry, 14(115).

Schulz, A., Stolz, T., Vincent, A., \& Krieger, T., Andersson, G., \& Berger, T. (2016). A sorrow shared is a sorrow halved? A three-arm randomized controlled trial comparing internetbased clinician-guided individual versus group treatment for social anxiety disorder. Behaviour Research and Therapy, 84, 14-26. doi: http://dx.doi.org/10.1016/j.brat.2016.07.001

Spek, V., Cuijpers, P., Nyklicek, I., Riper, H., Keyzer, J., \& Pop, V. (2007). Internet-based cognitive behaviour therapy for symptoms of depression and anxiety: A meta-analysis. Psychological Medicine, 37, 319-328. doi: 10.1017/S0033291706008944

Spence, S. H., Donovan, C. L., March, S., Gamble, A., Anderson, R., Prosser, S., . . Kenardy, J. (2008). Online CBT in the treatment of child and adolescent anxoety disorders: Issues in the development of BRAVE-ONLINE and two case illustrations. Behavior and Cognitive Therapy, 36, 411-430. doi: 10.1017/S135246580800444X

Stangier, U., Heidenreich, T., Berardi, A., Golbs, U., \& Hoyer, J. (1999). Die Erfassung sozialer Phobie durch die Social Interaction Anxiety Scale (SIAS) und die Social Phobia Scale (SPS). Zeitschrift für Klinische Psychologie, 28, 28-36. doi: 10.1026//0084-5345.28.1.28

Stangier, U., Heidenreich, T., \& Peitz, M. (2003). Soziale Phobien. Ein kognitivverhaltenstherapeutisches Behandlungsmanual. Weinheim: Beltz.

Tillfors, M., Furmark, T., Carlbring, P., \& Andersson, G. (2015). Risk profiles for poor treatment response in internet-delivered CBT in people with social anxiety disorder. Journal of Anxiety Disorders, 33, 103-109.

Titov, N., Andrews, G., Choi, I., Schwencke, G., \& Johnston, L. (2009). Randomized controlled trial of web-based treatment of social phobia without clinician guidance. Australian and New Zealand Journal of Psychiatry, 43, 913-919. 
A. Schulz, A. Vincent, \& T. Berger

Pragmatic Case Studies in Psychotherapy, http://pcsp.libraries.rutgers.edu

Volume 13, Module 3, Article 2, pp. 217-252, 11-11-17 [copyright by authors]

Table 1. Content of the Internet-Based Self-Help Program for SAD

(Berger, Boettcher, \& Caspar, 2013)

Session 1: Motivational enhancement

Session 2: Psychoeducation

Session 3: Cognitive restructuring

Session 4: Self-focused attention

Session 5: Behavioral experiments

Session 6: Summary and repetition

Session 7: Healthy lifestyle and problem solving

Session 8: Relapse prevention
Reasons to initiate change, definition of goals, recording of difficult social situations in an anxiety diary.

Introduction to Progressive Muscle Relaxation.

Information on SAD and its maintaining processes such as negative beliefs, self-focused attention, and safety behaviors.

Development of an individual model for SAD.

Identification and modification of dysfunctional assumptions using a thought record.

Various exercises to reduce self-focused attention, e.g., short behavioral experiments.

Planning and implementation of in vivo exposures.

Summary of the key elements of the treatment with emphasis on the importance of repeated practice (e.g. in vivo exposure).

Information on healthy lifestyle behavior (physical exercise and nutrition).

Development of problem solving skills.

Strategies for maintaining the acquired skills.

Preparation for possible relapse. 
A. Schulz, A. Vincent, \& T. Berger

Pragmatic Case Studies in Psychotherapy, http://pcsp.libraries.rutgers.edu

Volume 13, Module 3, Article 2, pp. 217-252, 11-11-17 [copyright by authors]

Table 2. Primary and Secondary Outcome Measures at Baseline

(Before Treatment), at Post-Treatment, and at Six-Month Follow-Up

DAYDREAMER

\begin{tabular}{|l|l|l|l|l|}
\hline Measure & $\begin{array}{l}\text { Clinical } \\
\text { Cut-Off }\end{array}$ & $\begin{array}{l}\text { Score at } \\
\text { Baseline }\end{array}$ & $\begin{array}{l}\text { Score at } \\
\text { Post-Treatment }\end{array}$ & $\begin{array}{l}\text { Score at Six- } \\
\text { Month Follow-Up }\end{array}$ \\
\hline $\begin{array}{l}\text { Social Phobia scale } \\
\text { (SPS) }\end{array}$ & 22 & 47 & $8^{*}$ & $9^{*}$ \\
\hline $\begin{array}{l}\text { Social Interaction } \\
\text { Anxiety Scale } \\
\text { (SIAS) }\end{array}$ & 33 & 69 & $28^{*}$ & $28^{*}$ \\
\hline $\begin{array}{l}\text { Beck Depression } \\
\text { Inventory (BDI-II) }\end{array}$ & $\#$ & 32 & $1^{*}$ & $1^{*}$ \\
\hline $\begin{array}{l}\text { Brief Symptom } \\
\text { Inventory (BSI) }\end{array}$ & -- & 2.09 & .28 & .25 \\
\hline $\begin{array}{l}\text { Inventory of } \\
\text { Interpersonal } \\
\text { Problems (IIP) }\end{array}$ & -- & 3.02 & 1.36 & 1.52 \\
\hline
\end{tabular}

NIGHT OWL

\begin{tabular}{|l|l|l|l|l|}
\hline Measure & $\begin{array}{l}\text { Clinical } \\
\text { Cut-Off }\end{array}$ & $\begin{array}{l}\text { Score at } \\
\text { Baseline }\end{array}$ & $\begin{array}{l}\text { Score at } \\
\text { Post-Treatment }\end{array}$ & $\begin{array}{l}\text { Score at Six- } \\
\text { Month Follow-Up }\end{array}$ \\
\hline $\begin{array}{l}\text { Social Phobia scale } \\
\text { (SPS) }\end{array}$ & 22 & 32 & 23 & $20^{*}$ \\
\hline $\begin{array}{l}\text { Social Interaction } \\
\text { Anxiety Scale } \\
\text { (SIAS) }\end{array}$ & 33 & 58 & 38 & 50 \\
\hline $\begin{array}{l}\text { Beck Depression } \\
\text { Inventory (BDI-II) }\end{array}$ & $\#$ & 8 & 2 & 14 \\
\hline $\begin{array}{l}\text { Brief Symptom } \\
\text { Inventory (BSI) }\end{array}$ & -- & 1 & .49 & 1.02 \\
\hline $\begin{array}{l}\text { Inventory of } \\
\text { Interpersonal } \\
\text { Problems (IIP) }\end{array}$ & -- & 2.6 & 2.3 & 2.0 \\
\hline
\end{tabular}

\# BDI clinical cut-off: 0-13: no or minimal depression; 14-19: mild depression; 20-28: moderate depression: 29-63: severe depression

* Below clinical cut-off 
Table 3. Excerpt of Daydreamer's Thought Diary

\begin{tabular}{|c|c|c|c|c|}
\hline Situation & $\begin{array}{l}\text { Thoughts and } \\
\text { worries }\end{array}$ & $\begin{array}{l}\text { Evidence that } \\
\text { supports/opposes the } \\
\text { thought }\end{array}$ & $\begin{array}{l}\text { Realistic } \\
\text { assessment }\end{array}$ & Consequence \\
\hline $\begin{array}{l}\text { A party } \\
\text { where I } \\
\text { don't know } \\
\text { a lot of } \\
\text { people }\end{array}$ & $\begin{array}{l}\text { Everybody will } \\
\text { think I'm weird } \\
\text { because I won't } \\
\text { dare to say } \\
\text { anything. They } \\
\text { will look at me } \\
\text { funny and avoid } \\
\text { me. }\end{array}$ & $\begin{array}{l}\text { Maybe people will } \\
\text { understand that someone } \\
\text { who is new doesn't talk } \\
\text { much. } \\
\text { Prior experience: I have } \\
\text { been to parties where I } \\
\text { felt good and found } \\
\text { someone to talk to, and } \\
\text { other parties where I felt } \\
\text { weird and out of place. }\end{array}$ & $\begin{array}{l}\text { True: } 50 \% \\
\text { Fear: } 5 / 10\end{array}$ & $\begin{array}{l}\text { Nobody will talk } \\
\text { to me and I won't } \\
\text { be invited the next } \\
\text { time. }\end{array}$ \\
\hline$\rightarrow$ & $\begin{array}{l}\text { Nobody will talk } \\
\text { to me and I won't } \\
\text { be invited the next } \\
\text { time. }\end{array}$ & $\begin{array}{l}\text { I've usually found } \\
\text { somebody to talk to. And } \\
\text { even if I don't that } \\
\text { doesn't mean that people } \\
\text { will dislike me. } \\
\text { My cousin is a good } \\
\text { friend and won't break } \\
\text { ties with me, no matter if } \\
\text { I do or don't speak to } \\
\text { people at the party. }\end{array}$ & $\begin{array}{l}\text { True: } 0 \% \\
\text { Fear: 0/10 }\end{array}$ & \\
\hline
\end{tabular}


A. Schulz, A. Vincent, \& T. Berger

Pragmatic Case Studies in Psychotherapy, http://pcsp. libraries.rutgers.edu

Volume 13, Module 3, Article 2, pp. 217-252, 11-11-17 [copyright by authors]

Table 4. Excerpt From Night Owl's Thought Diary

\begin{tabular}{|c|c|c|c|c|}
\hline Situation & $\begin{array}{l}\text { Thoughts and } \\
\text { worries }\end{array}$ & $\begin{array}{l}\text { Evidence that } \\
\text { supports/opposes the } \\
\text { thought }\end{array}$ & $\begin{array}{l}\text { Realistic } \\
\text { assessment }\end{array}$ & Consequence \\
\hline $\begin{array}{l}\text { At the } \\
\text { playground } \\
\text { with my } \\
\text { daughter, I } \\
\text { haven't seen } \\
\text { her for a } \\
\text { couple of } \\
\text { minutes and } \\
\text { don't know } \\
\text { where she } \\
\text { is. }\end{array}$ & $\begin{array}{l}\text { It's my fault if } \\
\text { something } \\
\text { happens to her or } \\
\text { if she's afraid. }\end{array}$ & $\begin{array}{l}\text { It has happened before } \\
\text { that I lost sight of her } \\
\text { somewhere, but nothing } \\
\text { bad has ever happened } \\
\text { and she never seemed } \\
\text { distraught when I found } \\
\text { her. } \\
\text { She's probably just } \\
\text { caught up in her playing. }\end{array}$ & $\begin{array}{l}\text { True: } 30 \% \\
\text { Fear: } 3 / 10\end{array}$ & $\begin{array}{l}\text { My daughter is } \\
\text { suffering; I failed } \\
\text { to watch over her, } \\
\text { people won't trust } \\
\text { me anymore. } \\
\text { I'll be even more } \\
\text { insecure in the } \\
\text { future. }\end{array}$ \\
\hline
\end{tabular}


A. Schulz, A. Vincent, \& T. Berger

Pragmatic Case Studies in Psychotherapy, http://pcsp.libraries.rutgers.edu

Volume 13, Module 3, Article 2, pp. 217-252, 11-11-17 [copyright by authors]

Table 5. The Clients’ Usage Data and Therapy Monitoring

\begin{tabular}{|l|l|c|l|}
\hline & Daydreamer & Night Owl & Average $^{1}$ \\
\hline Number of Modules completed & & & \\
\hline Total time logged on (hours) & 8 & 8 & $6.32(S D=2.16)$ \\
\hline Total number of diary entries & 36 & 34 & 16.5 \\
$\quad \begin{array}{l}\text { Relaxation } \\
\quad \text { Anxiety-provoking situations }\end{array}$ & 46 & 168 & 45 \\
$\quad \begin{array}{l}\text { Dysfunctional thoughts } \\
\text { Exposure exercises (Reality testing) }\end{array}$ & 14 & 39 & 14 \\
\hline Client messages sent & 11 & 57 & 14 \\
\hline Therapist messages sent & 6 & 33 & 9 \\
\hline $\begin{array}{l}\text { Client time spent in contact section } \\
\text { (hours) }\end{array}$ & 14 & 39 & 8 \\
\hline $\begin{array}{l}\text { Therapist time spent on guidance } \\
\text { (hours) }\end{array}$ & 6 & 11 & 6 \\
\hline
\end{tabular}

Note. ${ }^{1}$ refers to the average data of the clients in the individual guidance condition of the RCT. 
Daydreamer and Night Owl: Comparing Positive and Negative Outcome Cases in an Online, Clinician-Guided, Self-Help Intervention for Social Anxiety Disorder

A. Schulz, A. Vincent, \& T. Berger

Pragmatic Case Studies in Psychotherapy, http://pcsp.libraries.rutgers.edu

Volume 13, Module 3, Article 2, pp. 217-252, 11-11-17 [copyright by authors]

Figure 1. Course of Daydreamer's Symptoms on the Social Phobia Scale (SPS) and the Social Interaction Anxiety Scale (SIAS)

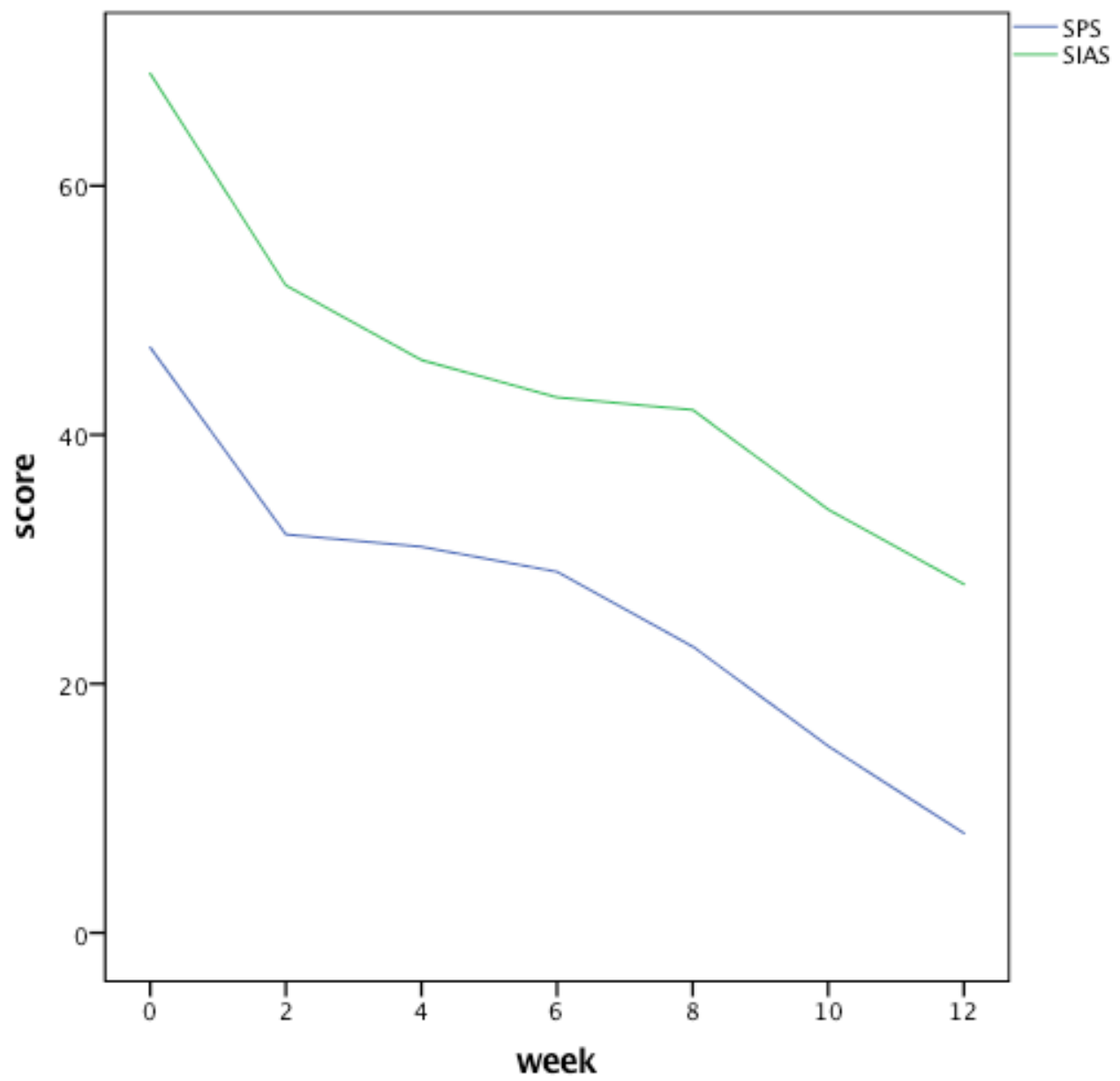


Daydreamer and Night Owl: Comparing Positive and Negative Outcome Cases in an Online, Clinician-Guided, Self-Help Intervention for Social Anxiety Disorder

A. Schulz, A. Vincent, \& T. Berger

Pragmatic Case Studies in Psychotherapy, http://pcsp.libraries.rutgers.edu

Volume 13, Module 3, Article 2, pp. 217-252, 11-11-17 [copyright by authors]

Figure 2. Course of Night Owl's Symptoms on the Social Phobia Scale (SPS) and the Social Interaction Anxiety Scale (SIAS)

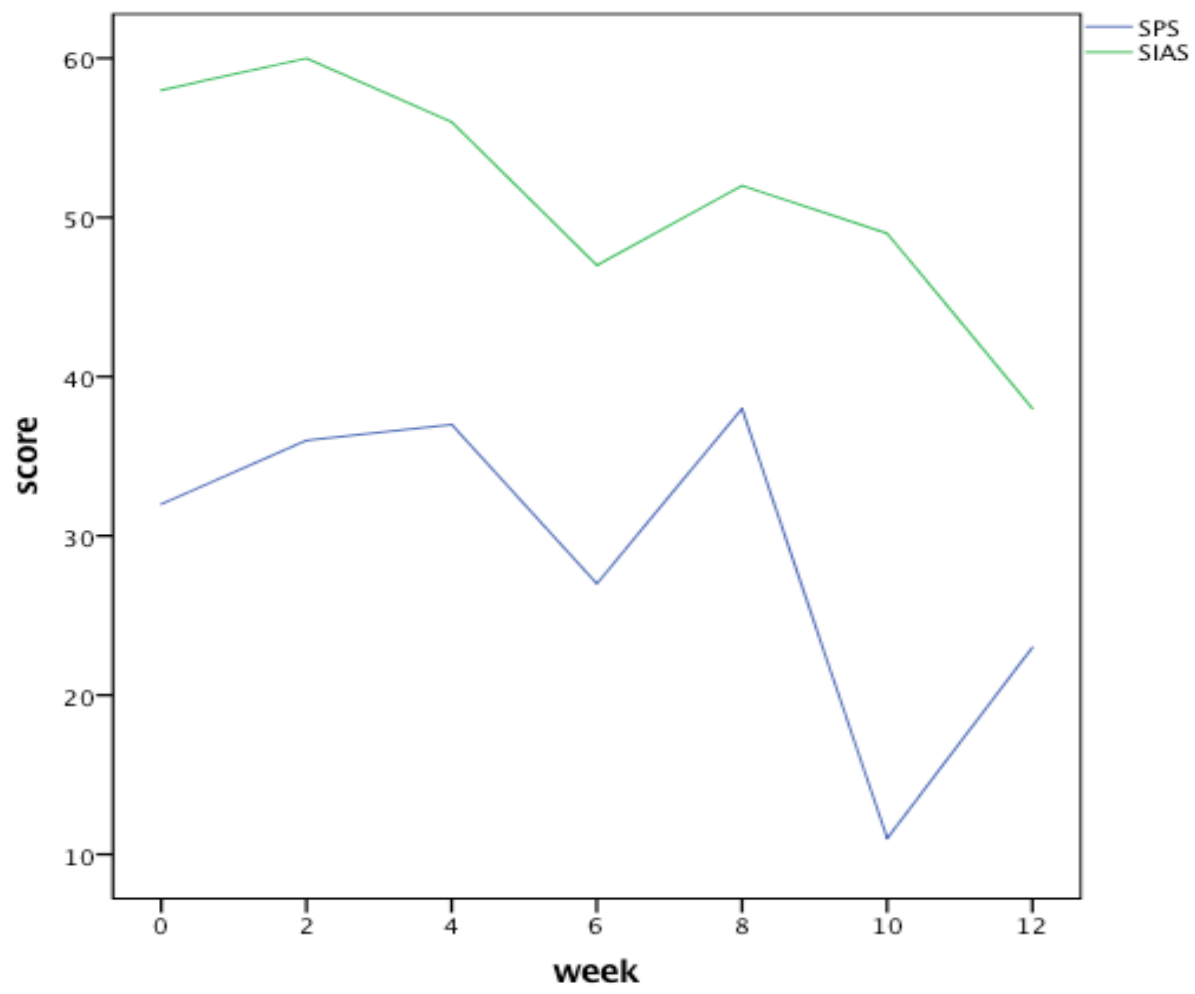

